\title{
Visualizing Ecosystems of Hype
}

\author{
Rahul C. Basole \\ Georgia Institute of Technology \\ basole@gatech.edu
}

\begin{abstract}
The emergence of new technologies is relentless. While there are many studies that have examined the transformative value, impact, and adoption, our understanding of the underlying ecosystem that shapes the emergence of these new technologies is surprisingly limited. Motivated by the call for pursuing contemporary information systems (IS) research that captures market realities and provides practical relevance, this study uses a data-driven visualization approach to map the ecosystem structure of emerging technologies. We use text mining to identify and extract relevant companies associated with 34 emerging technologies from publicly available data sources (business and technology news, press releases, industry briefings, analyst reports, and blogs) and then apply graph visualization techniques to reveal the corresponding ecosystem structure. We frame our analysis and discussion of these ecosystems in terms of the emerging technology's maturity stage and time to mainstream adoption. We illustrate our approach using visualizations of nine emerging technologies. We conclude with theoretical and practical implications and offer directions for future data-driven emerging technology ecosystem research.
\end{abstract}

\section{Introduction}

The emergence of new technologies is relentless [24]. In the last few years alone we have seen the introduction of many promising technological innovations including blockchain, 3D printing, cloud computing, Internet of Things, and conversational UI. Making sense of these continuous waves of innovation is challenging [33]. Some emerging technologies are truly transformative, enabling significant operational efficiencies and generating new ways of value creation [36]. However, many others never reach their touted potential and fail to meet expectations. Differentiating hype from actual potential is crucial to IT innovation decision making.

URI: http://hdl.handle.net/10125/50510

ISBN: 978-0-9981331-1-9

(CC BY-NC-ND 4.0)
One approach of understanding the maturity and value state of an IT innovation is to explore the broader context in which these technologies are created and used [37]. There is a growing consensus that new technological innovations are a result of a complex, collaborative and coopetitive web of interfirm activities. Yet our systematic understanding of the underlying ecosystem that shapes the emergence of these new technologies is limited. Who are the actors that participate in emerging technology ecosystems? What structural characteristics do emerging technology ecosystems exhibit? And how do these characteristics differ by the stage of maturity and years to mainstream adoption of an emerging technology?

We pursue these questions using a data-driven visual analytic approach. Specifically, we use the public narrative - mentions of the technology in a wide range of textual sources (business and technology news, press releases, industry briefings, analyst reports, and blogs) - as a source and probing lens to understand how firms, public and private, are associated and interconnected within each ecosystem. We begin by using text analytic techniques to identify and extract companies associated with emerging technologies identified by recent Gartner Hype Cycle reports and then apply graph visualization techniques to reveal the corresponding ecosystem structure. In doing so, we aim to uncover the structural characteristics of each ecosystem and, following suggestions by prior research [28], determine whether these characteristics differ by maturity stage and timeframe to mainstream adoption.

Our focus on understanding ecosystems of emerging technologies is motivated by the call for pursuing contemporary IS research. It has been argued that information systems (IS) research has a tendency to focus on well established, enduring, and foundational issues [30]. Consequently, practitioners are often unable to find the latest buzzwords, hot new trends, and recent technological developments in the academic literature. As a result academic research is often perceived as sluggish in capturing market realities and providing practical relevance.

Theoretically, our work builds and extends the literature on IT innovation and organizational learning by proposing the use of an ecosystemic lens of

\section{$\mathrm{H}_{\mathrm{I}}^{\mathrm{II}} \mathrm{CSS}$}


emerging technologies. Methodologically, we contribute to the IS field by combining computational techniques to identify and extract relevant information from large, unstructured datasets and visualize this data to reveal the interfirm ecosystem structure shaping emerging technologies. In doing so, we provide a complementary strategic intelligence approach which decision makers can use to assess the rapid, uncertain, and potentially ambiguous technology landscape.

The remainder of this study is organized as follows. Section 2 provides a background on the origin and characteristics of the hype cycle. Section 3 reviews relevant related work. Section 4 provides overview of our methodology. Section 5 presents and discusses the results. Section 6 concludes the study with theoretical and practical implications and opportunities for future research.

\section{Related work}

\subsection{Innovation and Hype Cycles}

The study of emerging technologies is extensive and a comprehensive review would be beyond the scope of this paper (see [11, 13, 15, 24, 29, 35, 36, 38]. Prominent topics have ranged from the underlying characteristics of the innovation and the adopters (e.g. [22]), the value and relevance of the innovation to individuals, organizations, and markets (e.g. [32]) to the process of diffusion (e.g. [16, 31]).

Almost every model depicts technology emergence and diffusion as a multi-phase phenomenon, in which decision makers become aware and interested in a technology, use and evaluate it on a trial-basis, and then either adopt or abandon it based on the experienced (or perceived) value the technology provides. Similarly, every technology experiences an upswing and downswing phase, driven by community learning, media hype, or vendor push. Indeed, some technologies experience rapid growth due to fads, fashion, and bandwagon effects (e.g. [1, 3, 29, 38].

Many economists and analysts have noted these technology "waves", and arguably one of the most prominent instrumentation is the "hype cycle". First introduced in 1995 by research advisory firm Gartner, the Hype Cycle is a graphical and conceptual depiction of a common pattern that arises with a new/emerging technology or innovation [25]. At a high level, hype cycles have been developed to help organizations track the maturity and future potential of technologies and decide which emerging technology to adopt and when [28]. The hype cycle characterizes the typical progression of innovation, from over-enthusiasm through a period of disillusionment to an eventual understanding of the innovation's relevance and role in a market or domain (see Figure 1). Each year Gartner creates nearly 100 hype cycles in different areas of technology, topic and industry.

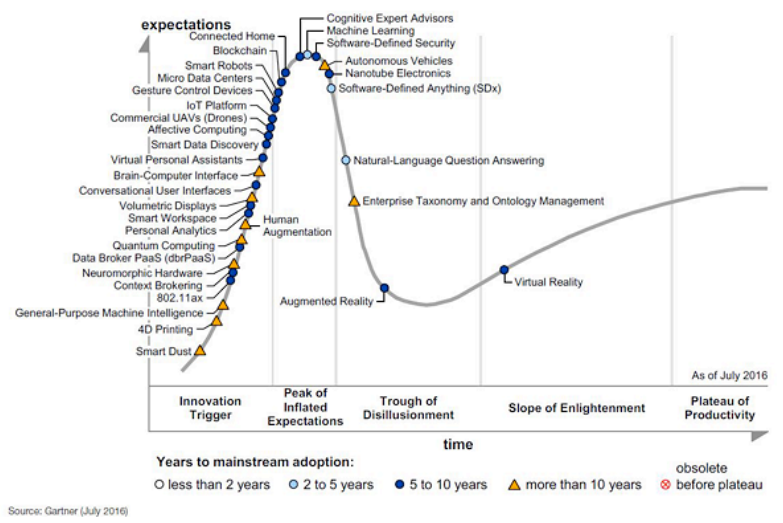

Figure 1. Emerging technology hype cycle example ${ }^{1}$

Based on the hype cycle, a technology passes through one of five stages on its path to broad assimilation and productivity. It begins with the Innovation Trigger (Stage I) when a breakthrough, public demonstration, product launch, or some other even generates press and industry interest in a technology innovation. In the Peak of Inflated Expectations stage (Stage II) a wave of "buzz" is built and expectation for a given technology rises above the current realized capabilities. In the Trough of Disillusionment stage (Stage III), impatience for results replaces the original excitement about the potential value. In this stage, problems with performance, slower-than-expected adoption, or a failure to deliver the appropriate financial returns lead to missed expectations and disillusionment sets in. In the Slope of Enlightenment stage (Stage IV) initial hurdles are overcome, and some adopters being to experience benefits and recommit efforts to move forward. During this stage, understanding grows about where and how a technology can be used. Finally, in the Plateau of Productivity stage (Stage V), real-world benefits of a technology is demonstrated and accepted, with an increasing number of organizations feeling comfortable with the greatly reduced levels of risk. During this stage, the technology experiences a sharp uptick in adoption (i.e. the "hockey stick") and assimilation accelerates rapidly as a result of the productive and useful value.

The $\mathrm{x}$-axis of the hype cycle thus captures "time" reflecting the fact that a particular technology will progress through each stage as time passes. The y-axis

\footnotetext{
${ }^{1}$ Source: http://www.gartner.com/newsroom/id/3412017
} 
is labeled "expectations" denoting how expectations (and thereby sentiment) surge and contract over time as a technology progresses, based on the market's assessment of its future expected value.

Not surprisingly, technologies do not move at a uniform speed through each of the stages. The speed at which technologies progress depends on many factors, including perceived/experienced value, market conditions, and competitive offerings. To capture the varying speeds, each technology thus also assessed relative to how long it will take to reach the Plateau of Productivity (i.e. mainstream adoption) from a technology's current position on the hype cycle. Five different speeds are identified ranging from less than two years, two to five years, five to 10 years, more than 10 years, and obsolete before it reaches the plateau (thus indicating it will either fail in the market or be overtaken by other competing technologies).

While hype cycles have proven to be valuable, they have also received a fair amount of criticism, including the fact that their design and development is somewhat simplistic and not scientifically guided, stage labeling is ambiguous and left to interpretation, merely comments on pre-existing trends, does not consider the speed at which technologies change, and fails to capture actual technology maturity levels (as for instance compared to the technology readiness levels). We do not deny these shortcomings, but rather argue that technologies identified in hype cycle reports do reflect an adequate broad sentiment of current trends. As such we believe hype cycles can provide a valuable foundation to identify relevant emerging technologies for analysis.

\subsection{Ecosystem analysis and visualization}

Technological innovations today do not emerge in isolation. To understand a firm's actions, choices, and outcomes, in particular in the context of emerging technologies, "an ecosystem perspective is neither necessary nor sufficient, but increasingly critical" due to the fundamentally changing nature of economic activities [2]. Similar to biological systems consisting of a variety of different species with symbiotic relationships, ecosystems are characterized as a complex set of multilateral ties between a wide range of stakeholders [19]. These stakeholders can include firms, customers, non-profit organizations, and government agencies [4].

The ecosystem metaphor for describing innovation activities and strategies is not new (see [26]) and has seen a particular significant growth in the IS and strategy literature. Examples of prior work has examined developer ecosystems (e.g. Linux), platform ecosystems (e.g. Apple vs. Google), and innovation ecosystems (e.g. Silicon Valley), just to name a few [5, $14,17,19,34,39]$. The primary focus of these studies has been on describing the structure and dynamics of these complex systems, including the role and positions actors assume, clusters that are formed, and how they evolve. The behavior of an ecosystem cannot be identified by inspection of the entities alone, but rather by the interaction of the entities. Ecosystems are highly dynamic, with entities entering and leaving, relationships formed, renewed, and deleted, and entity and relationship attributes changing constantly. It is this scale, complexity, and emergent dynamism that makes systematic understanding of ecosystems particularly challenging.

With the remarkable increase in open, sociallycurated, and proprietary data, however, there has been a greater opportunity to understand ecosystems using novel computational tools [7, 8, 9]. Indeed, novel visual analytic tools for understanding ecosystems are now available. Our study adopts an ecosystemic lens and leverages these solutions to provide insights into the emergence of new technologies.

\section{Methodology}

\subsection{Data}

Our study uses several secondary data sources to study the ecosystems of emerging technology hype. First, we use Gartner's Emerging Technology Hype Cycles $^{2}$ to identify relevant emerging innovations. As we discussed above, hype cycles represent the maturity, adoption and social application of specific technologies. We identified and extracted high-quality large images of Gartner's annual emerging technology hype cycles using a Google Image search ("Hype Cycle" and "Emerging Technologies" and "YYYY", where YYYY denoted the year of interest). For each of the images, we manually extracted and recorded the name of the emerging technology, the current stage, the maturity level (i.e. years to mainstream adoption), and the order number. We organized this data in a web-based spreadsheet for subsequent retrieval and analysis.

Second, we use ecoxight/pulse ${ }^{4}$, a visual ecosystem intelligence platform, powered by NorthernLight Millie, a competitive intelligence content platform, to identify relevant textual documents associated with each technology.

\footnotetext{
2 http://www.gartner.com/technology/research/methodologies/hypecycle.jsp

3 https://images.google.com/

4 https://ecoxight.com/pulse
} 
NorthernLight Millie provides web-based access to newswires, global business and technology news, IT industry reports as well as analyst reports and blogs. The portal contains a text analytics and meaning extraction engine developed specifically for market intelligence applications. We conducted two separate types of queries for each emerging technology to identify the relevant text corpora, namely in the fulltext and in title-only. The former query option provides a comprehensive narrative overview of the entity, while the latter provides a more focused lens. For each of the textual documents, a list of publicly-listed companies and privately-held ventures are identified.

We chose 2014-2016 5 as our study timeframe for several reasons. First, we wanted to make sure that we capture a wider set of emerging technologies for each hype cycle stage. Second, we wanted to track the progression of emerging technologies across stages. Lastly, and perhaps critically, we wanted to make sure that our timeframes across datasets aligned. Since NorthernLight Millie provides access to only the past three years of data, we focused our analysis to that timeframe.

\subsection{Construction of ecosystem graph}

ecoxight/pulse converts the co-occurrence data of these firms into a corresponding ecosystem network structure, with nodes depicting firms and edges between nodes representing the co-occurrence of the two firms in a given document. Edges are scaled (weighted) proportionally to the number of documents. We construct an ecosystem graph for each emerging technology. ecoxight/pulse uses a relevance-based caching strategy (high relevance documents are extracted first) to extract documents to optimize the construction of the network graph. Pending the number of documents for a given query, the data extraction and network construction process can be time consuming. On average, it takes 7-10 seconds to extract, mine, and process 100 documents (up to 10,000 documents). The total time can fluctuate pending the number of entities extracted. The process time increases significantly for very large datasets ( $>50,000$ documents). For instance, for our largest dataset of nearly 100,000 documents, the ecosystem graph construction time took nearly three hours. Our study uses several secondary data sources to study the ecosystems of emerging technology hype.

\footnotetext{
${ }^{5}$ We should note that emerging technology hype cycles are released in July of every year. At the time of submission, the 2017 hype cycle was not yet available.
}

\subsection{Metrics}

Following prior work on ecosystem analysis [6], we compute several important network-level metrics for each ecosystem, namely average degree, average weighted degree, network diameter, graph density, modularity, number of connected components, average clustering coefficient, and average path length. A more detailed explanation of these metrics can be found in [27].

\subsection{Visualization}

Visualizations are a fundamental component of human learning and understanding and a key step in transforming data to knowledge [12]. Visualization can be used to explore, interpret and communicate data and aid decision makers with overcoming cognitive limitations. By mapping data to visual encodings, visualizations of ecosystems make the "what, why, how, and who" explicit. Prior work has provided important novel and complementary insights into the structure, dynamics, and strategy of business ecosystems $[5,8,20]$.

We export the graph structure of each ecosystem from ecoxight/pulse in .gexf format for a more refined visualization and analysis in Gephi ${ }^{6}$. Gephi is an opensource software for visualizing and analyzing large network graphs [10]. Specifically, we use the forcedirected Yifan Hu multi-level layout algorithm [18]. A force-based layout is based on the idea that network entities are shaped by mechanical laws, assigning repulsive forces between nodes and attraction forces between endpoints of edges. The use of a force-based layout is particularly appealing when the motivating issue is to identify central or prominent nodes, peripheral actors, or clusters in an ecosystem. The Yifan $\mathrm{Hu}$ multilevel layout combines a force-directed model with a graph coarsening technique to reduce the complexity. We use the default initial parameter configuration. To ensure readability and aesthetics, we followed several visual design principles, including no node overlap and edge crossing minimization. In all our network visualization, node size is proportional to the firm's importance as measured by Betweenness centrality. To gain insight into the presence of subcommunities, we color encode nodes with the corresponding modularity class. We use a NoOverlap algorithm to space out nodes and address potential visual occlusion issues.

\footnotetext{
${ }^{6}$ http://www.gephi.org
} 


\section{Discussion of results}

Our analysis focused on all emerging technologies that appeared in the 2016 hype cycle report. More than half of these 34 emerging technologies are categorized in Stage I (19 technologies, 55.8\%) followed by Stage II (10 technologies, 29.4\%). Only very few technologies are in Stage III (4 technologies, 11.8\%) and Stage IV (1 technology, 2.9\%) and there are no technologies listed in Stage V, indicating a highly skewed hype cycle towards relatively new technologies. Broadly, these technologies can be classified as enabling transparent and immersive experiences (e.g., human augmentation, affective computing, brain-computer interface, augmented reality, virtual reality), accelerating the smart machine age (e.g., machine learning, smart robots, autonomous vhicles, drones, natural language question answering), and driving the platform revolutions (e.g., blockchain, IoT platform, software-defined security). Examining these technologies in terms of years to mainstream adoption, 3 technologies are in the near-timeframe (2-5 years), 20 technologies are in the mid-timeframe (510 ), and 11 are in the long timeframe (more than 10 years), indicating a balanced distribution.

Table 1 provides a summary of our text analytics and ecosystem analysis for each of the 34 emerging technologies. Column (A) indicates the number of unique documents identified for each emerging technology. The results show that the average number of documents is 11,297. The most number of documents extracted is for "virtual reality" with 95,783 , followed by "machine learning" with 77,436 , and "augmented reality" with 40,832. Not surprisingly, each of these technologies fall into the latter stages of the maturity cycle. Among Stage I (innovation trigger) technologies, "quantum computing" and "commercial drones" provide the most number of documents. In general, it can be observed that Stage I technologies receive significantly less coverage than Stage II technologies $(p<0.05)$. Column (B) presents results for title-only queries. Interestingly, one of the leading technologies mentioned in document titles is "blockchain", suggesting not only the relevance of the technology in general, but also the narrative prominence overall. When taking a 'years to mainstream adoption' lens, it can be seen that "autonomous vehicles" received the most attention in our data with 28,436 documents, followed by a relatively distant second "quantum computing". Among the more mid-term (5-10 years) technologies, the "connected home" received" the most attention.

Column (C) and (D) present the results of our entity extraction analysis, with number of companies and company edges shown respectively. The average number of companies found in each emerging technology ecosystem is 1,597 . However, the variance is quite large, with "virtual reality" $(7,006)$, "machine learning" (6,399) and "natural language question answering" $(5,168)$ ecosystems containing the most number of companies. This result confirms the general observation that many companies are operating or considering these technologies. The fewest number of companies identified were in "context brokering" and "volumetric displays"

Columns (E) through (M) present the results of our network analysis of each emerging technology ecosystem. The first network metric is the average degree (Column (E)). The average degree refers to the average number of links a firm in this ecosystem has to other firms. Across all ecosystems, the average degree is 4.030 , indicating that firms roughly are on average co-mentioned with four other companies. Another key network metric is the density of the ecosystem, which describes the ratio of links that exist in the network to the number of potential links that could be formed if all possible pairs of nodes were directly connected. The results indicate that the average density is 0.013 , suggesting an overall very low level of interconnectedness. A related metric to density is the average clustering coefficient, which captures the degree to which a firm's connections are co-mentioned with each other. The results indicate that the average clustering coefficient is 0.545 . Two other important ecosystem topology metrics are the network diameter and the average path length, which denote how "distant" nodes in the ecosystem are. The average path length is 6.156 suggesting that any two firms are on average six links removed from each other. The longest average path lengths can be found in Stage I emerging technologies (namely for "human augmentation" and for "IoT platform") suggesting that actors within these ecosystems are somewhat removed from each other.

Figure 2 presents a scatterplot of our text analytic results. Not surprisingly, we observe that there is a strong positive correlation between the number of documents and the number of companies extracted. Moreover, we can see that Stage I technologies tend to have significantly fewer companies (and documents) than Stage II-IV technologies. When considering "outlier" technologies we observe that there is a disproportionally larger number of documents than companies in the smart robots category, suggesting that there is significant coverage but relatively speaking there are fewer companies identified in this space. On the flip side, we see many more companies than documents for software-defined anything (SDx) area, suggesting that this technology may have not received as much attention yet, but many firms are associated with this emerging technology. 
Table 1. Summary of emerging technology ecosystem characteristics.

\begin{tabular}{|c|c|c|c|c|c|c|c|c|c|c|c|c|c|c|c|}
\hline Emerging Technology & $\begin{array}{c}\text { (A) } \\
\text { Stage }\end{array}$ & $\begin{array}{c}\text { (B) } \\
\text { Adopt }\end{array}$ & $\begin{array}{l}\text { (C) } \\
\text { Text }\end{array}$ & $\begin{array}{l}\text { (D) } \\
\text { Title }\end{array}$ & $\begin{array}{c}(\mathbf{E}) \\
\text { Ratio }\end{array}$ & $\begin{array}{c}(\mathbf{F}) \\
\text { Nodes }\end{array}$ & $\begin{array}{c}\text { (G) } \\
\text { Edges }\end{array}$ & $\begin{array}{l}\text { (H) } \\
\text { Deg }\end{array}$ & $\begin{array}{c}\text { (I) } \\
\operatorname{Deg}(w)\end{array}$ & $\begin{array}{c}(\mathbf{J}) \\
\text { Diam }\end{array}$ & $\begin{array}{l}\text { (K) } \\
\text { Dens }\end{array}$ & $\begin{array}{l}\text { (L) } \\
\text { Mod }\end{array}$ & $\begin{array}{c}\text { (M) } \\
\text { Comp }\end{array}$ & $\begin{array}{l}(\mathrm{N}) \\
\text { Clus }\end{array}$ & $\begin{array}{r}(0) \\
\text { APL }\end{array}$ \\
\hline Smart Dust & I & $>10$ & 120 & 16 & $13.3 \%$ & 114 & 150 & 2.632 & 3.333 & 10 & 0.023 & 0.745 & 34 & 0.440 & 3.721 \\
\hline 4D Printing* & I & $>10$ & 405 & 87 & $21.5 \%$ & 226 & 284 & 2.513 & 3.106 & 15 & 0.011 & 0.857 & 60 & 0.627 & 6.190 \\
\hline General-Purpose Machine Intelligence & I & $>10$ & 3,989 & 178 & $4.5 \%$ & 1,959 & 5,216 & 5.325 & 10.782 & 17 & 0.003 & 0.827 & 341 & 0.513 & 6.462 \\
\hline $802.11 \mathrm{ax}$ & I & $5-10$ & 243 & 45 & $18.5 \%$ & 219 & 375 & 3.425 & 5.507 & 15 & 0.016 & 0.796 & 34 & 0.549 & 6.404 \\
\hline Context Brokering & I & $5-10$ & 19 & 1 & $5.3 \%$ & 25 & 31 & 2.480 & 2.480 & 2 & 0.103 & 0.321 & 11 & 0.759 & 1.483 \\
\hline Neuromorphic Hardware & I & $>10$ & 291 & 79 & $27.1 \%$ & 273 & 476 & 3.487 & 5.187 & 17 & 0.013 & 0.871 & 57 & 0.562 & 6.924 \\
\hline Data Broker PaaS (dbrPaaS) & I & $5-10$ & 216 & 0 & $0.0 \%$ & 555 & 1,382 & 4.980 & 7.441 & 18 & 0.009 & 0.880 & 60 & 0.609 & 7.642 \\
\hline Quantum Computing* & I & $>10$ & 5,981 & 1,526 & $25.5 \%$ & 1,415 & 2,742 & 3.876 & 9.990 & 16 & 0.003 & 0.746 & 295 & 0.560 & 6.846 \\
\hline Human Augmentation* & I & $>10$ & 363 & 23 & $6.3 \%$ & 401 & 752 & 3.751 & 4.589 & 22 & 0.009 & 0.855 & 64 & 0.635 & 8.588 \\
\hline Personal Analytics & I & $5-10$ & 157 & 3 & $1.9 \%$ & 214 & 313 & 2.925 & 3.262 & 9 & 0.014 & 0.890 & 56 & 0.635 & 3.600 \\
\hline Smart Workspace & I & $5-10$ & 65 & 5 & $7.7 \%$ & 79 & 86 & 2.177 & 4.000 & 8 & 0.028 & 0.794 & 19 & 0.472 & 2.946 \\
\hline Volumetric Displays & I & $>10$ & 65 & 3 & $4.6 \%$ & 67 & 85 & 2.537 & 2.746 & 8 & 0.038 & 0.800 & 18 & 0.584 & 3.677 \\
\hline Conversational User Interfaces & I & $5-10$ & 283 & 7 & $2.5 \%$ & 308 & 498 & 3.234 & 5.487 & 17 & 0.011 & 0.805 & 75 & 0.567 & 6.634 \\
\hline Brain-Computer Interface* & I & $>10$ & 1,329 & 134 & $10.1 \%$ & 451 & 689 & 3.055 & 4.758 & 17 & 0.007 & 0.887 & 117 & 0.623 & 7.649 \\
\hline Virtual Personal Assistants & I & $5-10$ & 1,063 & 30 & $2.8 \%$ & 590 & 917 & 3.108 & 7.315 & 19 & 0.005 & 0.760 & 134 & 0.580 & 7.866 \\
\hline Smart Data Discovery & I & $5-10$ & 205 & 8 & $3.9 \%$ & 237 & 495 & 4.177 & 6.464 & 15 & 0.018 & 0.780 & 37 & 0.573 & 6.557 \\
\hline Affective Computing & I & $5-10$ & 754 & 17 & $2.3 \%$ & 400 & 606 & 3.030 & 4.980 & 19 & 0.008 & 0.832 & 106 & 0.589 & 7.370 \\
\hline Commercial UAVs (Drones) & I & $5-10$ & 5,394 & 631 & $11.7 \%$ & 1,527 & 3,814 & 4.995 & 9.597 & 18 & 0.003 & 0.776 & 232 & 0.547 & 6.389 \\
\hline IoT Platform* & I & $5-10$ & 1,111 & 105 & $9.5 \%$ & 916 & 1,786 & 3.900 & 6.555 & 18 & 0.004 & 0.859 & 147 & 0.570 & 7.929 \\
\hline Gesture Control Devices & II & $5-10$ & 2,212 & 9 & $0.4 \%$ & 798 & 1,269 & 3.180 & 9.376 & 17 & 0.004 & 0.784 & 203 & 0.526 & 6.884 \\
\hline Micro Data Centers & II & $5-10$ & 4,117 & 77 & $1.9 \%$ & 2,511 & 7,691 & 6.126 & 21.712 & 15 & 0.002 & 0.787 & 317 & 0.518 & 6.184 \\
\hline Smart Robots & II & $5-10$ & 24,264 & 63 & $0.3 \%$ & 534 & 897 & 3.360 & 5.251 & 16 & 0.006 & 0.836 & 110 & 0.585 & 6.931 \\
\hline Blockchain* & II & $5-10$ & 32,184 & 10,779 & $33.5 \%$ & 5,005 & 14,717 & 5.881 & 26.364 & 15 & 0.001 & 0.726 & 702 & 0.485 & 6.122 \\
\hline Connected Home & II & $5-10$ & 37,538 & 5,299 & $14.1 \%$ & 4,686 & 11,548 & 4.929 & 27.813 & 15 & 0.001 & 0.757 & 858 & 0.475 & 6.142 \\
\hline Cognitive Expert Advisors & II & $5-10$ & 719 & 0 & $0.0 \%$ & 1,241 & 3,467 & 5.587 & 9.046 & 17 & 0.005 & 0.869 & 140 & 0.560 & 7.132 \\
\hline Machine Learning & II & $2-5$ & 77,436 & 6,316 & $8.2 \%$ & 6,399 & 17,050 & 5.329 & 24.383 & 16 & 0.001 & 0.734 & 1,190 & 0.430 & 5.914 \\
\hline Software-Defined Security & II & $5-10$ & 348 & 33 & $9.5 \%$ & 336 & 616 & 3.667 & 6.446 & 14 & 0.011 & 0.812 & 44 & 0.498 & 6.272 \\
\hline Autonomous Vehicles* & II & $>10$ & 28,436 & 1,939 & $6.8 \%$ & 3,736 & 9,041 & 4.840 & 55.580 & 17 & 0.001 & 0.374 & 696 & 0.491 & 6.616 \\
\hline Nanotube Electronics* & II & $5-10$ & 2,027 & 18 & $0.9 \%$ & 725 & 1,309 & 3.611 & 5.189 & 16 & 0.005 & 0.867 & 168 & 0.546 & 7.059 \\
\hline Software-Defined Anything (SDx) & III & $2-5$ & 694 & 123 & $17.7 \%$ & 965 & 3,139 & 6.506 & 11.442 & 21 & 0.007 & 0.850 & 102 & 0.577 & 7.067 \\
\hline Natural-Language Question Answering & III & $2-5$ & 15,359 & 2 & $0.0 \%$ & 5,168 & 12,836 & 4.967 & 16.348 & 17 & 0.001 & 0.773 & 926 & 0.498 & 6.294 \\
\hline Enterprise Taxonomy \& Ontology Mgmt & III & $>10$ & 91 & 36 & $39.6 \%$ & 52 & 80 & 3.077 & 3.346 & 9 & 0.060 & 0.612 & 9 & 0.443 & 3.805 \\
\hline Augmented Reality* & III & $5-10$ & 40,832 & 3,767 & $9.2 \%$ & 5,150 & 13,075 & 5.078 & 27.616 & 18 & 0.001 & 0.624 & 957 & 0.457 & 5.973 \\
\hline Virtual Reality & IV & $5-10$ & 95,783 & 12,917 & $13.5 \%$ & 7,006 & 18,793 & 5.265 & 40.014 & 15 & 0.001 & 0.629 & 1,364 & 0.444 & 6.023 \\
\hline Mean & & & 11,297 & 1,302 & $9.8 \%$ & 1,597 & 4,007 & 4.030 & 11.691 & 15.24 & 0.013 & 0.768 & 285 & 0.545 & 6.156 \\
\hline Median & & & 909 & 41 & $7.3 \%$ & 573 & 1,093 & 3.709 & 6.510 & 16 & 0.007 & 0.795 & 114 & 0.555 & 6.433 \\
\hline Standard Deviation & & & 22489.26 & 3085.31 & 0.10 & 2048.44 & 5528.76 & 1.19 & 11.98 & 4.09 & 0.02 & 0.13 & 372.10 & 0.071 & 1.556 \\
\hline Min & & & & & $0.0 \%$ & 25 & 31 & 2.177 & 2.480 & 2 & 0.001 & 0.321 & 9 & 0.430 & 1.483 \\
\hline Max & & & 95,783 & 12,917 & $39.6 \%$ & 7,006 & 18,793 & 6.506 & 55.580 & 22 & 0.103 & 0.890 & 1,364 & 0.759 & 8.588 \\
\hline
\end{tabular}

Maturity Stages: $\quad$ Innovation Trigger (I) $\mid$ Peak of Inflated Expectations (II) | Through of Disillusionment (III) | Slope of Enlightenment (IV) | Plateau of Productivity (V)

Ecosystem Metrics: $\quad$ Avg. Degree (Deg) $\mid$ Avg. Weighted Degree (Deg(w)) $\mid$ Network Diameter (Diam) $\mid$ Modularity (mod) $\mid$ Connected Components (Comp) $\mid$ Avg. Clustering Coefficient (Clus) $\mid$ Avg. Path Length (APL)

*Ecosystem visualizations for emerging technologies provided in Figures 3a-i. 


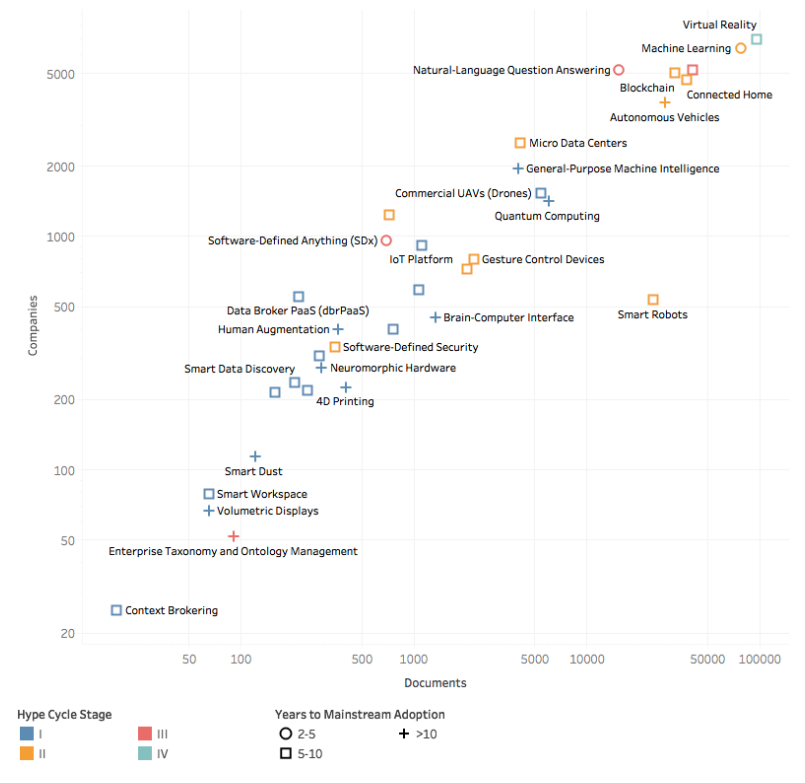

Figure 2. Scatterplot of text entity extraction results (Number of documents vs. number of companies).

While statistical summaries of network structure are valuable, visual representations of ecosystems can provide many additional important and complementary insights. Given space constraints, we limit our visual representations to subset of emerging technology ecosystems. Figure $3 \mathrm{a}-\mathrm{i}$ provides nine illustrative examples (i.e. 4D printing, human augmentation, brain-computer interface, nanotube electronics, IoT platform, quantum computing, autonomous vehicles, blockchain, and augmented reality), ordered by ecosystem size (from small to large). The examples we have chosen represent a mix of emerging technologies across innovation stages and years to maturity of adoption. The purpose of these visual illustrations is to highlight some of the similarities and differences in topological structure of different ecosystems. We acknowledge that higher resolution images and node labels would have helped with readability, but due to space constraints we were unable to do so. An anonymized URL to the images is available upon request.

The visualizations clearly reveal the wide range of ecosystem size, with augmented reality, blockchain and autonomous vehicles being significantly larger than, for instance, 4D printing and brain-computer interfaces. The difference in size can be explained in part by the broader relevance and impact of the former technologies to a wide range of companies and industries. It can also be explained in part by the maturity of the technology and thereby greater interest by the community.
Another striking observation across all ecosystems, including those not shown, is the clear demarcation of a core-periphery structure. A core-periphery network indicates that there are potentially disconnected activities occurring in an ecosystem, with the majority of firms connected to a core and a smaller set of companies operating on the periphery. It should be noted that while companies may be operating at the periphery it does not mean that they are not important to the ecosystem, but rather indicates that there may be different foci areas within each ecosystem.

Each ecosystem is color-encoded by modularity class. Modularity captures the degree of subcommunities present within ecosystems. Across all emerging technology ecosystems we find a range of sub-communities are present, ranging from a few to many. This, too, reflects the potential presence of specialized activities (i.e., collaborations, coopetitions, etc.) within these ecosystems.

\section{Concluding remarks}

This study uses a data-driven visualization approach to map the interfirm structure of emerging technology ecosystems. Using text analytic techniques, we extract relevant companies from publicly available data sources and then apply graph visualization techniques to reveal the corresponding ecosystem structure. Our results show that there are some commonalities but also significant differences between emerging technology ecosystems in terms of stage of maturity and timeframe to mainstream adoption. All ecosystems tend to exhibit a significant core-periphery structure, suggesting that there are diverse sets of collaborative and coopetitive activities happening across all ecosystems.

The implications of our study are multifold. First, emerging technologies are the result of a complex web of interfirm activities. In order for decision makers to cope with the speed, uncertainty, and ambiguity of the technology landscape, novel strategic sense-making tools are needed. Our data-driven visualization approach provides one such solution. Second, our results show that emerging technology ecosystems are highly variegated in nature, potentially consisting of different activity centers and foci. Thus, an examination of emerging technologies should take a wider view and consider all the diverse interfirm collaboration that may exist. Third, none of these technologies are truly independent. The growth and trajectory of these technologies is co-dependent on others. Thus, it would be valuable to consider developing macro-perspectives to see how these technologies in fact fit together. Each of these avenues present exciting avenues for future research. 


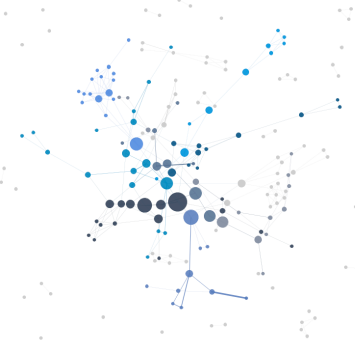

(a) 4D printing $(n=221)$

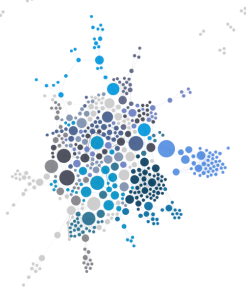

(d) Nanotube electronics $(n=725)$

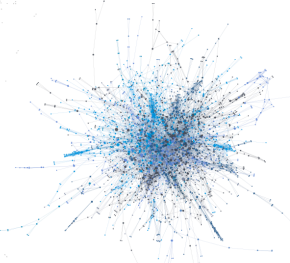

(g) Autonomous vehicles $(n=3,736)$

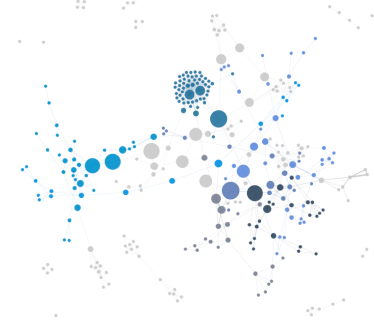

(b) Human augmentation $(n=401)$

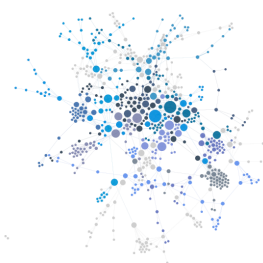

(e) IoT platform $(n=916)$

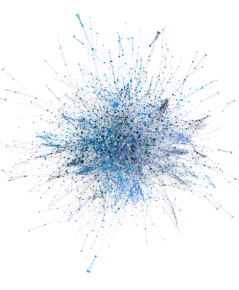

(h) Blockchain $(n=5,005)$

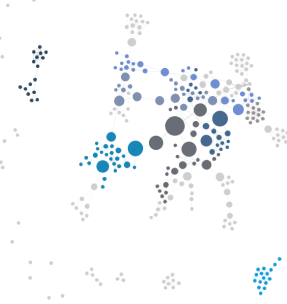

(c) Brain-computer interface $(n=451)$

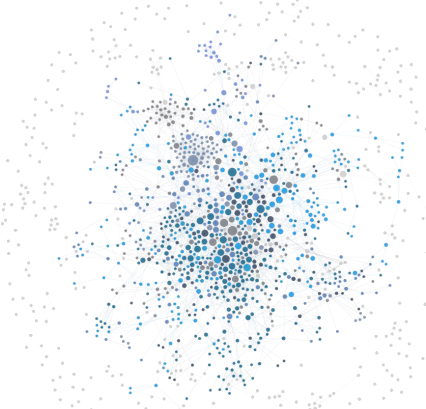

(f) Quantum computing $(n=1,415)$

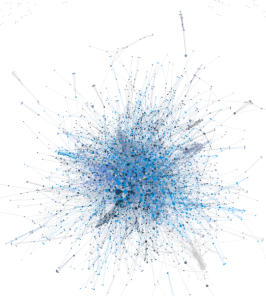

(i) Augmented reality $(n=5,150)$

Figure 3. Examples of emerging technology ecosystems (Nodes sized by Betweenness centrality; colored by modularity class). 


\section{References}

[1] Abrahamson, E. (1991). Managerial fads and fashions: The diffusion and rejection of innovations. Academy of Management Review, 16(3), 586-612.

[2] Adner, R. (2017). Ecosystem as Structure: An Actionable Construct for Strategy. Journal of Management, 43(1), 39-58.

[3] Baskerville, R. L., \& Myers, M. D. (2009). Fashion waves in information systems research and practice. MIS Quarterly, 647-662.

[4] Basole, R. C., \& Rouse, W. B. (2008). Complexity of service value networks: Conceptualization and empirical investigation. IBM Systems Journal, 47(1), 53-70.

[5] Basole, R. C. (2009). Visualization of interfirm relations in a converging mobile ecosystem. Journal of information Technology, 24(2), 144-159.

[6] Basole, R. C., Russell, M. G., Huhtamäki, J., Rubens, N., Still, K., \& Park, H. (2015). Understanding business ecosystem dynamics: a data-driven approach. ACM Transactions on Management Information Systems (TMIS), 6(2), 6.

[7] Basole, R. C. (2014). Visual business ecosystem intelligence: Lessons from the field. IEEE Computer Graphics and Applications, 34(5), 26-34.

[8] Basole, R. C., Clear, T., Hu, M., Mehrotra, H., \& Stasko, J. (2013). Understanding interfirm relationships in business ecosystems with interactive visualization. IEEE Transactions on Visualization and Computer Graphics, 19(12), 2526-2535.

[9] Basole, R. C., Huhtamäki, J., Still, K., \& Russell, M G. (2016). Visual decision support for business ecosystem analysis. Expert Systems with Applications, $65,271-282$

[10] Bastian, M., Heymann, S., \& Jacomy, M. (2009). Gephi: An open source software for exploring and manipulating networks. International Conference on Web and Social Media, 8, 361-362.

[11] Benbasat, I., \& Moore, G. C. (1992, January). Development of measures for studying emerging technologies. In System Sciences, 1992. Proceedings of the Twenty-Fifth Hawaii International Conference on (Vol. 4, pp. 315-324). IEEE.

[12] Card, S. K., Mackinlay, J. D., \& Shneiderman, B. (1999). Readings in information visualization: using vision to think. Morgan Kaufmann.

[13] Carr, N. G. (2003). IT doesn't matter. Havard Business Review, 305.
[14] Ceccagnoli, M., Forman, C., Huang, P., \& Wu, D. J. (2012). Cocreation of value in a platform ecosystem: the case of enterprise software. MIS Quarterly, 36(1), 263-290.

[15] Cegielski, C. G., Reithel, B. J., \& Rebman, C. M. (2005). Emerging information technologies: Developing a timely IT strategy. Communications of the ACM, 48(8), 113-117.

[16] Cooper, R. B., \& Zmud, R. W. (1990). Information technology implementation research: a technological diffusion approach. Management Science, 36(2), 123139.

[17] Evans, P. C., \& Basole, R. C. (2016). Revealing the API ecosystem and enterprise strategy via visual analytics. Communications of the ACM, 59(2), 26-28.

[18] Hu, Y. (2005). Efficient, high-quality force-directed graph drawing. Mathematica Journal, 10(1), 37-71.

[19] Iansiti, M., \& Levien, R. (2004). The keystone advantage: what the new dynamics of business ecosystems mean for strategy, innovation, and sustainability. Harvard Business Press.

[20] Iyer, B. R., \& Basole, R. C. (2016). Visualization to understand ecosystems. Communications of the ACM, 59(11), 27-30.

[21] Iyer, B., Lee, C. H., \& Venkatraman, N. (2006). Managing in a" small world ecosystem": Lessons from the software sector. California Management Review, $48(3), 28-47$.

[22] Jeyaraj, A., Rottman, J. W., \& Lacity, M. C. (2006). A review of the predictors, linkages, and biases in IT innovation adoption research. Journal of Information Technology, 21(1), 1-23.

[23] Keegan B, Gergle D, Contractor N (2013) Hot off the Wiki: Structures and dynamics of Wikipedia's coverage of breaking news events. American Behavioral Scientist 57(5), 595-622.

[24] Lee, A. (2000). Researchable directions for ERP and other new information technologies. MIS Quarterly, 24(1), III

[25] Linden, A., \& Fenn, J. (2003). Understanding Gartner's hype cycles. Strategic Analysis Report No R20-1971. Gartner, Inc.

[26] Moore, J. F. (1996). The death of competition: leadership and strategy in the age of business ecosystems. HarperCollins Publishers.

[27] Newman, M. E. (2003). The structure and function of complex networks. SIAM review, 45(2), 167-256. 
[28] O'Leary, D. E. (2008). Gartner's hype cycle and information system research issues. International Journal of Accounting Information Systems, 9(4), 240252.

[29] Ramiller, N. C. (2006, August). Hype! Toward A Theory of Exaggeration In Information Technology Innovation. In Academy of Management Proceedings (Vol. 2006, No. 1, pp. A1-A6). Academy of Management.

[30] Ramiller, N. C., Swanson, F. B., \& Wang, P. (2008). Research directions in information systems: toward an institutional ecology. Journal of the Association for Information Systems, 9(1), 1.

[31] Rogers, E. M. (2010). Diffusion of innovations. Simon and Schuster.

[32] Swanson, E. B. (1994). Information systems innovation among organizations. Management science, 40(9), 1069-1092.

[33] Swanson, E. B. (2012). The manager's guide to IT innovation waves. MIT Sloan Management Review, 53(2), 75 .
[34] Tiwana, A., Konsynski, B., \& Bush, A. A. (2010). Research commentary-Platform evolution: Coevolution of platform architecture, governance, and environmental dynamics. Information Systems Research, 21(4), 675-687.

[35] Van Lente, H., Spitters, C., \& Peine, A. (2013). Comparing technological hype cycles: Towards a theory. Technological Forecasting and Social Change, $80(8), 1615-1628$

[36] Venkatraman, V. (2017). The Digital Matrix: New Rules for Business Transformation Through Technology. Greystone Books.

[37] Wang, P., \& Ramiller, N. C. (2009). Community learning in information technology innovation. MIS Quarterly, 709-734.

[38] Wang, P. (2010). Chasing the hottest IT: effects of information technology fashion on organizations. MIS Quarterly, 34(1), 63-85.

[39] Yoo, Y., Henfridsson, O., \& Lyytinen, K. (2010). Research commentary-the new organizing logic of digital innovation: an agenda for information systems research. Information systems research, 21(4), 724735 . 